\title{
Two-component systems of the myxobacteria: structure, diversity and evolutionary relationships
}

Correspondence

David E. Whitworth

d.e.whitworth@warwick.ac.uk

dew@aber.ac.uk

Received 26 September 2007

Revised 28 October 2007

Accepted 13 November 2007
David E. Whitworth ${ }^{1,2}$ and Peter J. A. Cock ${ }^{3}$

\author{
${ }^{1}$ Department of Biological Sciences, University of Warwick, Gibbet Hill Road, Coventry CV4 7AL, \\ UK \\ ${ }^{2}$ Institute of Biological Sciences, Cledwyn Building, Aberystwyth University, \\ Ceredigion SY23 3DD, UK \\ ${ }^{3}$ MOAC Doctoral Training Centre, University of Warwick, Gibbet Hill Road, Coventry CV4 7AL, UK
}

\begin{abstract}
Two-component systems (TCSs) are a large family of signalling pathways characterized by the successive transfer of phosphoryl groups between the histidine and aspartate residues of paired histidine kinase and response regulator proteins. With the availability of genome sequences for four genera of myxobacteria it has become possible to assess the genomic complements of myxobacterial TCS genes and to characterize features of their organization and evolutionary heritage. In this study we have compiled lists of the TCS genes within myxobacterial genomes and characterized their domain architecture, gene organization and evolutionary relationships. In order to provide an appropriate context for our conclusions, where possible we have compared myxobacterial TCSs with those found in 316 other completely sequenced bacteria. Myxobacteria have the largest number of TCSs of any organisms. An unusually low proportion of TCS genes are paired in myxobacterial genomes, and myxobacterial histidine kinases also seem to sense internal signals to an unusual degree. Phylogenetic evidence has allowed us to suggest homologous relationships of proteins across the myxobacteria, and it appears that myxobacterial TCS evolution has been dominated by duplications, gene rearrangements and changes in sensory domain complements. The systematic classification of the TCS proteins of the myxobacteria presented here should also provide a framework for future experimental studies on twocomponent regulation in these organisms.
\end{abstract}

\section{INTRODUCTION}

Myxobacteria are members of the Deltaproteobacteria and form an important part of the soil community (Dawid, 2000). They are copious producers of antibiotics and other biologically active secondary metabolites (Gerth et al., 2003). However, the most striking behaviours of the myxobacteria are social: they exhibit co-ordinated motility, predating other members of the soil microfauna, and they possess a communal response to starvation. Under nutrient limitation a population of cells aggregates, forming a multicellular fruiting body within which cells differentiate into myxospores (for recent reviews see Kaiser, 2004; Whitworth,

Abbreviations: CDS, coding sequence; HK, histidine kinase; HY, hybrid kinase; $\mathrm{P}$, phosphotransfer protein; RR, response regulator; TCS, twocomponent system.

Three supplementary tables, of Stigmatella aurantiaca ORFs containing transmitter/receiver domains not identified as CDSs in GenBank, bacterial genomes surveyed and TCS proteins from the four myxobacterial genomes, and three supplementary figures showing phylogenetic trees for myxobacterial receiver domains, transmitter domains and Hpt domains are available with the online version of this paper.
2007). Genome sequences have become available for four myxobacteria, including the model myxobacterium Myxococcus xanthus DK1622 (Goldman et al., 2006). Sorangium cellulosum So ce56 and Stigmatella aurantiaca DW4/3-1 are both capable of forming fruiting bodies (Schneiker et al., 2007; Pradella et al., 2002; Neumann et al., 1993), while Anaeromyxobacter dehalogenans 2CP$\mathrm{C}$ exhibits an unusual degree of metabolic versatility (Sanford et al., 2002). A second Anaeromyxobacter genome (Anaeromyxobacter sp. Fw109-5) has recently become available, but was not included in this study. To date, more than 40 two-component system (TCS) proteins have been experimentally characterized and implicated in motility and/ or development in the model organism $M$. xanthus (Whitworth \& Cock, 2007). Sequence analysis shows that the genome sequences of myxobacteria contain an unparalleled number of TCS genes, making them important model organisms for studies of complexity in TCS signalling.

The first component in a typical TCS is a sensor kinase comprising an $\mathrm{N}$-terminal input domain and a C-terminal transmitter domain. The second TCS component is a response regulator, which usually possesses an $\mathrm{N}$-terminal 
receiver domain and a C-terminal output domain. Sensor kinase input domains are capable of sensing changes in their environment and, under appropriate conditions, trigger the activation of the transmitter domain. Activated transmitter domains then autophosphorylate on a conserved histidine residue. The phosphorylated transmitter domain is capable of binding to the response regulator receiver domain and in the resulting complex the phosphoryl group is transferred to an aspartate residue within the receiver domain. Phosphorylation of the response regulator alters the activity of the output domain, resulting in an appropriate response to the initial environmental change (for recent reviews see Bijlsma \& Groisman, 2003; West \& Stock, 2001). Often, the response regulator output domain has DNA-binding activity and phosphorylation of the response regulator leads to changes in DNA-binding activity and therefore gene expression.

More complex versions of TCSs exist with multiple phosphotransfer reactions. Phosphorelays are relatively common examples where there is successive transfer of phosphoryl groups from a transmitter domain to a receiver domain, to a His-containing phosphotransfer domain (usually a Hpt domain) and finally onto a second receiver domain (Appleby et al., 1996). The four TCS domains involved can be found on separate polypeptides (for example, the sporulation phosphorelay of Bacillus subtilis: Hoch \& Varughese, 2001) or as part of multi-domain proteins (for example, ArcBA of Escherichia coli: PeñaSandoval et al., 2005).

Input and output domains are diverse in sequence due to the variety of different stimuli to which TCSs respond and the different responses they elicit. However, transmitter and receiver domains retain high levels of sequence conservation, reflecting their common functions in the phosphotransfer scheme (Parkinson \& Kofoid, 1992). Typically the genes for sensor kinases are found adjacent to the genes for their partner response regulators. However, many 'orphaned' TCS genes are isolated in the genome, while others lie in clusters of many TCS genes. In such cases, the partner proteins and signalling pathways of the encoded TCS proteins are unclear.

Of the TCS proteins described in the model myxobacterium $M$. xanthus, most are involved with the regulation of development or motility (Whitworth \& Cock, 2007). However, the high proportion of orphan TCS genes in $M$. xanthus has hindered the description of entire TCS pathways. Orphan proteins with defined phenotypes include the sensor kinase SdeK and the response regulators ActA, ActB, Nla4, Nla6, FruA, FrzS and FrzZ, which all have roles in regulating motility and/or development (Pollack \& Singer, 2001; Gronewald \& Kaiser, 2001; Caberoy et al., 2003; Ellehauge et al. 1998; Ward et al., 2000; Trudeau et al., 1996). The partner signalling proteins of these orphan proteins remain to be identified.

Here we present a survey of the TCS genes encoded by the four available myxobacterial genomes. In addition to describing major features of the TCSs we provide an analysis of their evolutionary heritage, enabling identification of paralogous and orthologous relationships.

\section{METHODS}

Identification of TCS genes. TCS genes were identified using an automated procedure as described previously (Cock \& Whitworth, 2007a). Myxobacterial genomes surveyed were Anaeromyxobacter dehalogenans 2CP-C (NC_007760), Myxococcus xanthus DK1622 (NC_008095), Sorangium cellullosum So ce56 (EMBL accession number AM746676), and Stigmatella aurantiaca DW4/3-1 (AAMD01000000). We also identified 12 ORFs encoding receiver and transmitter domains in the St. aurantiaca genome (downloaded from http://www.tigr.org) that had not been identified as coding sequences (CDSs) in the GenBank accession. We have given these ORFs CDS designations STIAU_X\#\#\# and provided the translated ORF sequences as supplementary information (see Supplementary Table S1, available with the online version of this paper). In addition TCS complements of 316 completed bacterial genomes were surveyed (these genomes are listed in Supplementary Table S2, available with the online version of this paper).

Characterization of input/output domains, transmembrane helices and TCS gene organization. An automated assessment of input and output domains was undertaken by performing RPS-BLAST searches of all TCS CDSs against the Pfam database (Sonnhammer et al., 1997) with an expectation value cut-off of $10^{-4}$. Many domains in addition to transmitter and receiver domains were identified in this manner, and where possible these were classified manually as input or output domains. The presence/absence of transmembrane helices was assessed using TMHMM v2.0 (Sonnhammer et al. 1998). The presence of DNAbinding domains was determined by RPS-BLAST hits to a manually compiled list of PFAM domains (pfam00126, 00165, 00196, 00249, 00440, 00447, 00486, 01022, 01381, 02954, 04397, 04545 and 04967) using an expectation value cut-off of $10^{-4}$. Gene organization of TCS genes was determined using a classification scheme based on proximity to other TCS genes. If a TCS gene was separated from other TCS genes by $>5000$ bp it was considered to be orphan; paired TCSs were defined as two TCS genes on the same strand of DNA and separated by $<100 \mathrm{bp}$, encoding a total of one transmitter and one receiver domain. Any other gene organization was defined as complex, including gene clusters encoding multiple TCS genes containing more than one transmitter and/or more than one receiver domain. Orphan hybrid kinases were considered to be orphans regardless of the number of transmitter and receiver domains they encoded. The St. aurantiaca genome is incomplete and therefore TCS genes close $(<3 \mathrm{~kb})$ to the ends of contigs were annotated as having an uncertain gene organization.

Phylogenetic analysis. Multiple sequence alignments were generated for transmitter and receiver domains using CLUSTAL W (Thompson et al., 1994) with default parameter settings. Phylogenetic trees were constructed with 1000 bootstraps using the neighbourjoining algorithm implemented in QuickJoin (Mailund \& Pedersen, 2004), and 100 bootstraps using a parsimony algorithm, with PHYLIP (Felsenstein, 1989).

\section{RESULTS}

\section{Myxobacteria have extremely large numbers of TCSs}

Complete genome sequences are publicly available for three myxobacteria, M. xanthus DK1622 (9.1 Mbp), So. cellulo- 
sum So ce56 (13.0 Mbp) and A. dehalogenans 2CP-C $(5.0 \mathrm{Mbp})$. In addition, a partial genome sequence is available for St. aurantiaca DW4/3-1. This sequence consists of 61 scaffolds comprising a total of $10.1 \mathrm{Mbp}$ of DNA. An automated screen was employed to extract TCS genes from the myxobacterial genome sequences and from 316 other completely sequenced bacterial genomes in GenBank (ftp://ftp.ncbi.nlm.nih.gov/genomes/) as described previously (Cock \& Whitworth, 2007a). Putative TCS genes were then classified as response regulators (RRs; proteins encoding receiver domains but no transmitter domains), histidine kinases (HKs; transmitter domains but no receiver domains), hybrid kinases (HYs; transmitter and receiver domains) or phosphotransfer proteins $[\mathrm{Ps}$; Hpt or HisKA domains but no histidine ATPase (HATPase) or receiver domains].

Fig. 1 displays the number of TCS genes found in an organism against the genome size of that organism, for 320 bacteria (a similar distribution is seen when considering the number of receiver/transmitter domains; data not shown). At one extreme of the distribution are three of the myxobacterial genomes, with the largest numbers of TCS genes and receiver/transmitter domains of any organisms (Fig. 1). M. xanthus possesses 278 TCS proteins (337 receiver/transmitter domains), comprising $134 \mathrm{RR}, 99 \mathrm{HK}$, $41 \mathrm{HY}$ and $4 \mathrm{P}$ proteins. So. cellulosum encodes 267 TCS proteins (335 receiver/transmitter domains), consisting of 119 RRs, $98 \mathrm{HKs}, 49 \mathrm{HYs}$ and $1 \mathrm{P}$. St. aurantiaca has 326 TCS proteins (421 receiver and transmitter domains), 137 RRs, 120 HKs, 66 HYs and 3 Ps. The smallest myxobacterial genome (A. dehalogenans) has the lower numbers of TCS proteins (174 proteins, with 195 receiver and transmitter domains), including $92 \mathrm{RRs}, 65 \mathrm{HKs}$ and 17 HYs, but still more than most organisms of comparable genome size (the mean number of TCS genes for bacteria

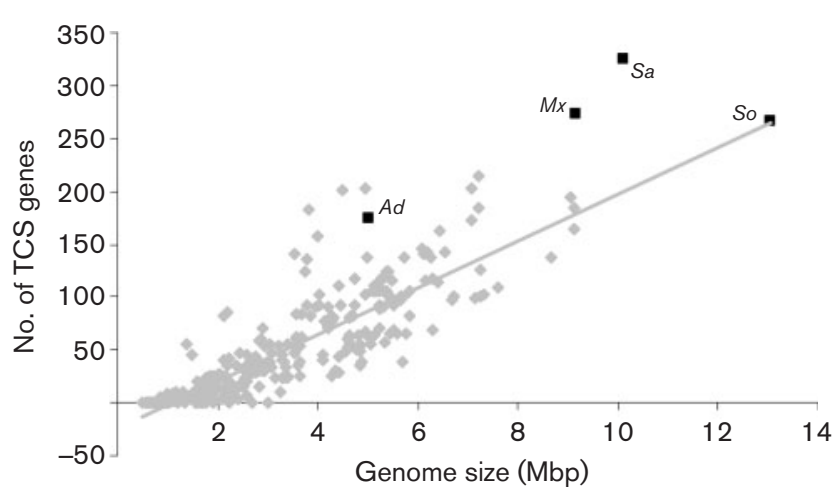

Fig. 1. Numbers of TCS genes found in bacterial genomes, as a function of genome size. Data from four myxobacteria (black) and 316 other bacteria (grey) are shown. A linear trendline is presented for non-myxobacterial organisms. Myxobacteria have significantly more TCS genes than would be predicted from their genome size. Ad, Anaeromyxobacter dehalogenans; Mx, Myxococcus xanthus; Sa, Stigmatella aurantiaca; So, Sorangium cellulosum. with genome sizes between 4 and $6 \mathrm{Mbp}$ is $81.7 \pm 35.3$ ). Lists of the myxobacterial TCS proteins, with descriptions of their genetic and domain architecture and whether they are predicted to possess transmembrane helices, are available as supplementary information (see Supplementary Table S3 available with the online version of this paper).

For most bacteria there is an approximately linear relationship between the number of encoded TCS proteins and genome size (Fig. 1). Organisms that have small numbers of TCSs for their genome size appear to live in relatively constant environments, as observed previously (Galperin, 2005), and include many pathogens. Several bacteria (mostly Delta/Epsilonproteobacteria) possess many more TCSs than most organisms of similar genome size, for example Wolinella succinogenes DSM 1740 (83 TCS genes, $2.1 \mathrm{Mbp}$ ), Desulfovibrio vulgaris Hildenborough (136 TCS genes, 3.8 Mbp), Geobacter sulfurreducens PCA (183 TCS genes, $3.8 \mathrm{Mbp}$ ), Nostoc sp. PCC 7120 (215 TCS genes, 7.2 Mbp) 'Dechloromonas aromatica' RCB (201 TCS genes, $4.5 \mathrm{Mbp}$ ) and the myxobacteria (Fig. 1). It has been proposed that the number of TCS genes within genomes correlates more with sophistication of lifestyle than with variability of environment (see Galperin, 2005 for a more involved discussion), a suggestion supported by the behavioural complexity of the myxobacteria.

For nearly all organisms (including the myxobacteria) there was a constant receiver domain: transmitter domain ratio $(\sim 3: 2)$, which was independent of genome size or the number of TCSs within each genome (data not shown). This suggests that in most organisms there are more divergent (one HK phosphorylating $>1 \mathrm{RR}$ ) than convergent (one RR being phosphorylated by $>1 \mathrm{HK}$ ) TCS pathways. For those organisms with $\geqslant 50$ TCS genes no examples were found with a receiver:transmitter ratio $>1.9$, while only nine had a receiver:transmitter ratio $<1.0$ (three Bacillus strains, three Bacteroides strains, Methanosarcina acetivorans, Haloarcula marismortui and Salinibacter ruber).

\section{A large proportion of myxobacterial TCS genes are orphaned or in complex clusters}

Typical TCSs consist of paired HK and RR genes (often translationally coupled). However, TCS genes can be found either isolated in the genome (orphaned) or in complex gene clusters. An orphan or complex organization may be indicative of multiple partnerships between TCS proteins (for example CheA, CheY and CheB of E. coli are found in a complex gene cluster, and the HK CheA phosphorylates both $\mathrm{CheB}$ and $\mathrm{CheY}$, while the proteins of the sporulation phosphorelay of Bacillus subtilis are orphaned). We classified the gene organization of all TCS proteins in our lists as either paired, orphan or complex (see Methods). The numbers and proportions of orphan, paired and complex systems varied widely between genomes. For organisms with $>50$ TCS genes, the proportion of paired 


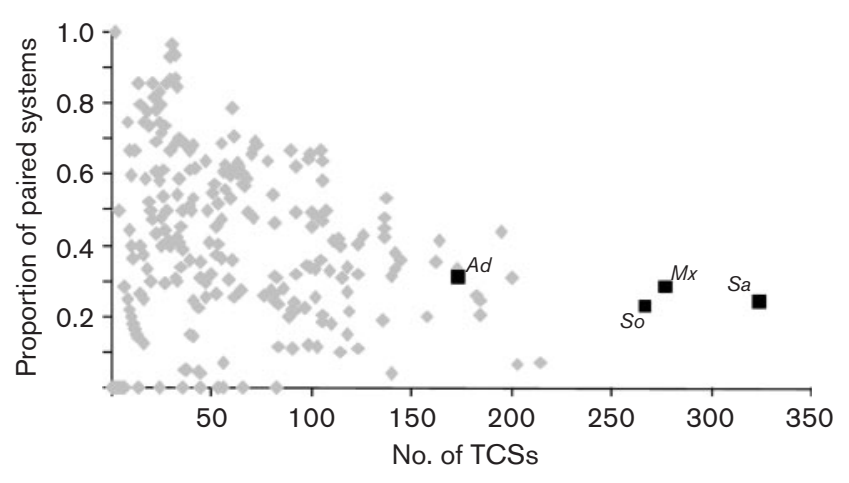

Fig. 2. Proportion of paired TCS genes within a genome with respect to the total number of TCS genes within that genome. Data plotted for four myxobacteria (black) and 316 other bacteria (grey). Organisms with larger numbers of TCS genes tend to have a smaller proportion of paired TCS genes. Ad, Anaeromyxobacter dehalogenans; Mx, Myxococcus xanthus; Sa, Stigmatella aurantiaca; So, Sorangium cellulosum.

TCS genes varied between 0.8 (Clostridium tetani E88) and 0.0 [two Cyanobacteria (Yellowstone A and B), Haloarcula marismortui and Methanosarcina acetivorans]. A plot of the proportion of paired TCS genes against the number of TCS genes in a genome (Fig. 2) demonstrates a reduced proportion of paired TCS genes as the number of TCS genes increases (compensated for by larger proportions of orphaned genes and complex gene clusters). This observation suggests that with increasing numbers of TCS genes comes increased communication between proteins. Table 1 provides a summary of the TCS gene organization of myxobacteria and, for comparison, the model organisms Escherichia coli, Bacillus subtilis, Streptomyces coelicolor and Synechocystis sp. PCC 6803. Compared to the model organisms, the myxobacteria have an unexpectedly large number of TCS genes within complex gene clusters, even when compensating for their larger numbers of TCSs.

Five TCS gene clusters of $M$. xanthus are particularly complex, encoding at least two histidine-containing phosphotransfer domains (transmitter and Hpt domains) and at least three receiver domains (Fig. 3). One of these clusters (redCDEF) has been characterized and shown to regulate the timing of multicellular development (Higgs et al., 2005); however, the role of the other clusters has yet to be determined. These gene clusters are currently being investigated experimentally in our laboratory.

\section{An unexpectedly large proportion of myxobacterial kinases are predicted to be cytoplasmic}

As a general rule the presence of transmembrane helices in a HK or HY would suggest a transmembrane localization and imply in turn that the kinase was responding to an extracytoplasmic stimulus. Conversely, soluble kinases would be expected to sense changes to the internal milieu. We assessed what proportion of sensor kinases possess predicted transmembrane helices and thus would be expected to respond to extracellular stimuli (see Methods). Fig. 4 shows how the number of sensor kinases (both HK and $\mathrm{HY}$ ) predicted to be transmembrane varies as a function of the total number of sensor kinases within a genome. For all bacteria with $\geqslant 10$ sensor kinases, the mean proportion of transmembrane kinases is $\sim 71.7 \%( \pm 20.2 \%)$, while the numbers are much lower for the myxobacteria. The large numbers of cytoplasmic HKs may explain the large proportion of HYs in myxobacterial genomes (Cock \& Whitworth, 2007b). Other organisms displaying unexpectedly low numbers of transmembrane sensor kinases include Nostoc sp. (39 of 133, $29 \%$ ), Anabaena variabilis (38 of 128, $30 \%)$, Methanosarcina acetivorans (10 of 49, $20 \%$ ), Haloarcula marismortui (14 of 58, 24\%) and Methanospirillum hungatei (24 of 76, 32\%). Presumably these organisms and the myxobacteria predominantly assess their environment by monitoring changes in their internal state. Within the myxobacterial lineage, the absolute number of transmembrane sensor kinases does not increase significantly with increasing genomic complement of TCSs, suggesting that the different myxobacteria respond to a similar number of extracellular factors (Fig. 4).

Table 1. Organization of the TCS genes of four myxobacteria and four model bacteria

TCS gene organizations are classified as paired, orphan or complex, as described in the text. Because the Stigmatella aurantiaca genome comprises 492 separate contigs an 'uncertain' gene organization indicates genes found towards the ends of contigs. Myxa, Myxococcus xanthus DK1622; Soce, Sorangium cellulosum So ce56; Stau, Stigmatella aurantiaca DW4/3-1; Ande, Anaeromyxobacter dehalogenans 2CP-C; Esco, Escherichia coli; Basu, Bacillus subtilis (str. 168), Stco, Streptomyces coelicolor A3(2); Syn. 6803, Synechocystis sp. PCC 6803.

\begin{tabular}{|lcccccccc|}
\hline Gene organization & Myxa & Soce & Stau & Ande & Esco & Basu $\dagger$ & Stco $\ddagger$ & Syn. 6803§ \\
\hline Paired & 80 & 62 & 78 & 56 & 44 & 60 & 112 & 22 \\
Complex & 93 & 91 & 97 & 44 & 9 & 0 & 25 & 55 \\
Orphan & 105 & 114 & 121 & 74 & 9 & 10 & 52 \\
Uncertain & - & - & 30 & - & - & - & - \\
Total & 278 & 267 & 326 & 174 & 62 & 70 & 192 & 86 \\
\hline
\end{tabular}

*, $\dagger, \ddagger$, §Numbers derived from Mizuno (1997), Fabret et al. (1999), Hutchings et al., (2004) and Mizuno et al. (1996), respectively. 

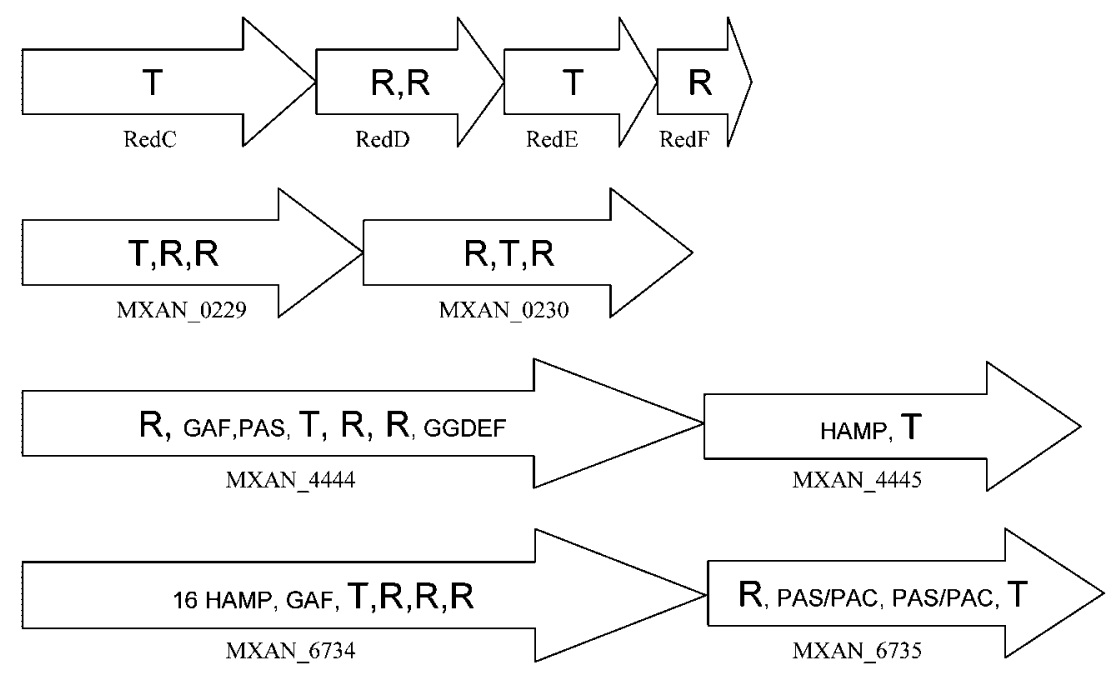

\begin{abstract}
Fig. 3. Complex TCS gene clusters of Myxococcus xanthus. Genes are represented as arrows, with the domain architecture of the predicted protein product as text within each arrow (presumed input/output domains are in small type). Arrow length is proportional to gene length. Each cluster contains at least two histidine-containing phosphotransfer domains ( $\mathrm{T}$ or $\mathrm{Hpt}$ ) and at least three receiver domains (R).
\end{abstract}

\section{TCS input and output domains are unevenly distributed across bacteria}

Bacterial predicted proteomes were assessed for the presence of conserved domains by searching against the Pfam database (see Methods). The most common Pfam domain was the ABC transporter (pfam00005), with 21364 such domains identified. The second most common domain was the receiver domain (pfam00072, 10171 cases), while the third most frequent domain was the HATPase domain (pfam02518, 8834 cases). Amongst the set of TCS proteins the most common non-phosphotransfer domain was the DNA-binding domain Trans_reg_C

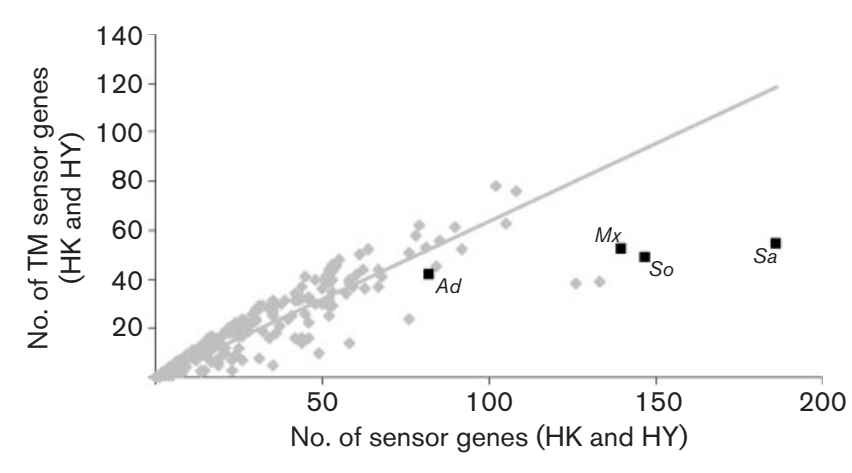

Fig. 4. Number of sensor kinases (HKs and HYs) predicted to be transmembrane, as a function of the total number of sensor kinases. Data plotted for four myxobacteria (black) and 316 other bacteria (grey). For all bacteria with $\geqslant 10$ sensor kinases, the mean proportion of transmembrane kinases is $\sim 71.7 \%( \pm 20.2)$, while the myxobacteria have substantially fewer transmembrane sensor kinases. Ad, Anaeromyxobacter dehalogenans; Mx, Myxococcus xanthus; Sa, Stigmatella aurantiaca; So, Sorangium cellulosum. (pfam00486, 2807 cases), which is found for example in PhoB and OmpR. Ten output domains (eight DNAbinding associated domains [Trans_reg_C, GerE, Sigma70_r4, HTH_Fis, HTH_10, Sigma54_activator/ AAA_ATPase, LytTR and HTH_AraC], CheB methylesterase and GGDEF domains) and five input domains (PAS, PAC, GAF, HAMP and CheW domains) were each found in $>200$ cases amongst the bacterial TCS proteins. The percentages of TCS proteins of the 320 bacteria containing these 15 common domains are presented in Table 2.

The distribution of the 15 common domains among the bacteria was assessed by comparing the numbers of a particular domain found in one organism's TCS proteins as a proportion of the total number of such domains found in the TCS proteins of all 320 bacteria, restricting analysis to genomes with $\geqslant 100$ TCS proteins ( 57 organisms). Organisms that encoded $>5 \%$ of all TCS proteins containing a particular domain are listed in Table 3. St. aurantiaca was found to contain large numbers of TCS proteins containing GAF domains. The cyanobacteria Nostoc and Anabaena also possessed large numbers of GAF domains, while Bacteroides thetaiotaomicron contained exceptionally large numbers of $\mathrm{HTH}$ _AraC domains. GAF domains are found in phytochromes and cGMP phosphodiesterases in addition to sensor proteins, but in the vast majority of cases the signals sensed by GAF domains are unknown. The proportion of the TCS proteins of an organism that contained each domain was also determined. In three cases $>40 \%$ of the TCS protein complement of an organism was found to contain a particular domain. Anabaena variabilis and Methanospirillum hungatei each possessed a large proportion of PAC domain-containing TCSs (43\% and $64 \%$ respectively, 88 and 89 cases), while Methanospirillum 
Table 2. Abundant input and output domains: percentage of TCS proteins that contain particularly common input and output domains (found in $>1 \%$ of all TCS proteins of 320 bacteria)

\begin{tabular}{|lc|}
\hline Domain & \% of TCSs \\
\hline Input domains & \\
PAS & 16.7 \\
HAMP & 15.7 \\
PAC & 11.7 \\
GAF & 5.4 \\
CheW & 2.5 \\
Output domains & \\
Trans_reg_C & 17.1 \\
GerE & 8.0 \\
Sigma70_r4 & 6.2 \\
HTH_8 & 5.0 \\
Sigma54_activator & 4.7 \\
GGDEF & 1.9 \\
HTH_10 & 1.7 \\
LytTR & 1.6 \\
HTH_AraC & 1.6 \\
CheB_Me-esterase & 1.3 \\
\hline
\end{tabular}

hungatei also possessed a large proportion of PAS domaincontaining TCSs (92\%, 129 cases). PAS and PAC domains are often associated with one another within proteins and act as signal sensor domains for a wide range of stimuli. Although the unexpectedly large numbers of certain accessory domains within the TCS proteins of specific organisms is intriguing, we have not been able to correlate the presence of those domains with particular physiological behaviours.

\section{Classification of myxobacterial response regulators}

Response regulators can be classified according to their domain architecture (Galperin, 2006). Most examples comprise an $\mathrm{N}$-terminal receiver domain and a C-terminal output domain, although more complicated geometries exist. Table 4 groups RRs by domain architecture for the myxobacterial proteins and those of Escherichia coli, Bacillus subtilis and Synechocystis sp. PCC 6803. RR families

Table 3. Abundant input and output domains: organisms that contain large numbers of particular input/output domains (>5\% of all TCS proteins containing those domains, from 320 bacteria)

\begin{tabular}{|llcc|}
\hline Domain & \multicolumn{1}{c}{ Organism } & $\begin{array}{c}\text { \% of all } \\
\text { TCSs }\end{array}$ & $\begin{array}{c}\text { Domain- } \\
\text { containing TCSs }\end{array}$ \\
\hline GAF & St. aurantiaca & 5.5 & 49 \\
GAF & Nostoc sp. & 7.3 & 65 \\
GAF & Anabaena variabilis & 7.8 & 69 \\
HTH_AraC Bacteriodes thetaiotaomicron & 16.7 & 43 \\
\hline
\end{tabular}

are named after well-characterized proteins where possible, or by domain architecture (see Table 4).

Myxobacterial RRs exhibit unusually large numbers of AAA_ATPase,HTH_Fis domains as found in NtrC homologues. Additionally they possess a large number of RRs that consist entirely of a single receiver domain, resembling CheY of E. coli. CheY homologues are involved in chemotactic regulation (phosphorylated by CheA homologues) and possess single receiver domains. CheA homologues are easily identified by the presence of an $\mathrm{Hpt}$ domain within their transmitter domains, and in most organisms cheA is encoded in the proximity of cheY. The proximity of a receiver-only RR gene to a cheA homologue enables us to classify receiver-only proteins as CheY homologues or not (a division that is supported by phylogenetic evidence; our unpublished data). In the myxobacteria the vast majority of receiver-only RRs are not CheY homologues (Table 4). Presumably they effect regulation by protein-protein contacts, act to modulate phosphoryl transfer to effector RRs, or participate in phosphorelays. The only other organisms which possess large numbers of receiver-only RRs are cyanobacteria, particularly Nostoc sp. PCC 7120 and Anabaena variabilis.

Over-represented RR families (even compensating for the large number of RRs in myxobacterial genomes) also include the CheY, CheB, PleD, RpfG, CyaB and K,R,Cyc families (Table 4). Several RR families appear to be unique to myxobacteria, including the FrzZ family of proteins, which contain tandem receiver domains, and the R,STAS family, which contain C-terminal anti-anti-sigma factor/ sulfate transport domains. The only common RR family that is under-represented in myxobacterial genomes is the NarL family, which includes the important regulator of fruiting FruA (Ellehauge et al., 1998).

\section{Myxobacterial histidine kinase input domains}

A variety of common input domains can be found in HKs and HYs, typically N-terminal to the transmitter domain. Table 5 lists the numbers of HKs and HYs in myxobacterial genomes that possess HAMP, GAF and/or PAS/PAC input domains. Also described are the numbers of CheA homologues, characterized by Hpt-containing transmitter domains and CheW domains.

Relatively uncommon input domains are also found amongst the myxobacterial TCS kinases (Table 6). Sixteen of the HKs and HYs of So. cellulosum (nine from St. aurantiaca and one from M. xanthus) possess Ser/Thr kinase domains (Table 6), implying an unusual degree of integration of Ser/Thr kinase and TCS signalling networks. The three myxobacteria containing Ser/Thr kinase input domains also contain HKs with FHA (forkhead-associated) domains implicated in transducing signals from Ser/Thr kinases (Jelsbak et al., 2005). All four myxobacteria contain HKs with input domains associated with regulation of potassium uptake/efflux (Kdp and KefB domains), three 
Table 4. Classification of myxobacterial response regulators by domain architecture

R, receiver domain; T, transmitter domain; K, Ser/Thr kinase domain; O, output domain; Myxa, Myxococcus xanthus DK1622; Soce, Sorangium cellulosum So ce56; Stau, Stigmatella aurantiaca DW4/3-1; Ande, Anaeromyxobacter dehalogenans 2CP-C; Esco, Escherichia coli; Basu, Bacillus subtilis (str. 168); Syn. 6803, Synechocystis sp. PCC 6803.

\begin{tabular}{|c|c|c|c|c|c|c|c|c|c|}
\hline Family & Domains & Myxa & Soce & Stau & Ande & Total (myxobacteria) & $\operatorname{Esco}^{\star}$ & Basu $\dagger$ & Syn. 6803‡ \\
\hline CheY & $\mathrm{R}$ & 8 & 7 & 9 & 7 & 31 & 1 & 1 & \\
\hline PhoB & R,Trans_reg_C & 11 & 11 & 10 & 5 & 37 & 14 & 14 & 10 \\
\hline NtrC & R,AAA_ATPase,HTH_Fis & 30 & 22 & 20 & 27 & 99 & 4 & - & - \\
\hline NarL & R,HTH_LuxR & 5 & 3 & 5 & 3 & 16 & 7 & 9 & 7 \\
\hline CheB & $\mathrm{R}, \mathrm{CheB}$ & 6 & 2 & 9 & 6 & 23 & 1 & - & - \\
\hline $\mathrm{R}$ & $\mathrm{R}$ & 46 & 51 & 60 & 31 & 188 & - & 2 & 7 \\
\hline FrzZ & $\mathrm{R}, \mathrm{R}$ & 4 & $4(1 \S)$ & 5 & 2 & 15 & - & - & - \\
\hline R,STAS & R,STAS & - & $4(1 \mid I)$ & - & - & 4 & - & - & - \\
\hline PleD & R,GGDEF & $8(2 \mid I)$ & 1 & 5 & $4(111)$ & 18 & - & - & - \\
\hline RpfG & $\mathrm{R}, \mathrm{HDc}$ & 3 & $1(1 \mid I)$ & 2 & 2 & 8 & - & - & - \\
\hline СуаB & R,СуаA & 1 & 2 & 1 & - & 4 & - & - & - \\
\hline $\mathrm{K}, \mathrm{R}, \mathrm{Cyc}$ & S/T_Kin,R,CyaA & 1 & 3 & 3 & - & 7 & - & - & - \\
\hline $\mathrm{RO}$ & $\mathrm{R}, \mathrm{O}$ (DNA-binding) & 4 & 4 & 2 & 1 & 11 & 2 & 3 & 1 \\
\hline $\mathrm{RO}$ & R,O (other) & 4 & 2 & 2 & 2 & 10 & 3 & 4 & 7 \\
\hline OR & $\mathrm{O}, \mathrm{R}$ & 2 & 1 & 3 & 2 & 8 & - & - & 6 \\
\hline ORO & $\mathrm{O}, \mathrm{R}, \mathrm{O}$ & - & 1 & 1 & - & 2 & - & - & - \\
\hline RHRRO & $\mathrm{R}, \mathrm{H}, \mathrm{R}, \mathrm{R}, \mathrm{GGDEF}$ & 1 & - & - & - & 1 & - & - & - \\
\hline Total & & 134 & 119 & 137 & 92 & 482 & 32 & 33 & 38 \\
\hline
\end{tabular}

$\star, \dagger, \ddagger$ Numbers derived from Mizuno (1997), Fabret et al. (1999) and Mizuno et al. (1996), respectively.

§Protein contains an additional Hpt domain.

IIProteins contain an additional GAF domain.

myxobacterial HYs possess paired CheB,CheR input domains, and three HKs possess PutP domains for $\mathrm{Na}^{+} /$ proline symport. Both $M$. xanthus and St. aurantiaca possess HKs with CAP-effector domains (CAP-EDs), which are implicated in binding cyclic nucleotides, St. aurantiaca possesses two HKs with phytochrome input domains, while intriguingly a $\mathrm{HK}$ of $A$. dehalogenans has an $\mathrm{N}$-terminal globin-coupled-receptor domain.

\section{Myxobacterial hybrid kinases have diverse and complicated domain architectures}

HYs can be classified according to the number and order of their transmitter and receiver domains (Table 7). The most common domain architectures consist of single receiver and transmitter domains (125 of 173); however, 48 HYs have multiple transmitter $(\mathrm{T})$ and/or receiver (R) domains.

Table 5. HK and HY input domains: numbers of myxobacterial HKs and HYs containing PAS, GAF or HAMP domains

Myxa, Myxococcus xanthus DK1622; Soce, Sorangium cellulosum So ce56; Stau, Stigmatella aurantiaca DW4/3-1; Ande, Anaeromyxobacter dehalogenans 2CP-C. Numbers of CheA homologues are also presented (characterized by a CheW input domain).

\begin{tabular}{|c|c|c|c|c|c|c|c|c|c|}
\hline \multirow[t]{2}{*}{ Input } & \multicolumn{4}{|c|}{ HKs } & \multicolumn{4}{|c|}{ HYs } & \multirow[t]{2}{*}{ Total } \\
\hline & Myxa & Soce & Stau & Ande & Myxa & Soce & Stau & Ande & \\
\hline PAS & 18 & 8 & 21 & 10 & 11 & 14 & 18 & 3 & 103 \\
\hline GAF & 7 & 13 & 23 & 3 & 3 & 2 & 11 & - & 62 \\
\hline HAMP & 12 & 19 & 13 & 12 & - & 8 & 3 & 1 & 68 \\
\hline PAS,GAF & 9 & 7 & 12 & 2 & - & 10 & 3 & - & 43 \\
\hline GAF,HAMP & - & - & - & - & 2 & 2 & - & - & 4 \\
\hline PAS,HAMP & 1 & 1 & - & - & - & - & - & - & 2 \\
\hline HAMP,CheA & - & 1 & - & - & - & - & - & - & 1 \\
\hline CheA & 3 & 5 & 5 & 5 & 5 & 1 & 4 & 2 & 30 \\
\hline None/other & 49 & 44 & 46 & 33 & 20 & 12 & 27 & 11 & 242 \\
\hline Total & 99 & 98 & 120 & 65 & 41 & 49 & 66 & 17 & 555 \\
\hline
\end{tabular}


Table 6. HK and HY input domains: unusual input domains found in myxobacterial kinases

Myxa, Myxococcus xanthus DK1622; Soce, Sorangium cellulosum So ce56; Stau, Stigmatella aurantiaca DW4/3-1; Ande, Anaeromyxobacter dehalogenans 2CP-C.

\begin{tabular}{|lcccc|}
\hline Input & Myxa & Soce & Stau & Ande \\
\hline S/T_Kinase & 1 & 16 & 9 & - \\
CAP-ED & 1 & - & 1 & - \\
Kdp & 1 & 1 & - & 1 \\
KefB & - & - & 1 & - \\
PhnD & - & - & 1 & - \\
CheB/CheR & - & 1 & 1 & 1 \\
PutP & 1 & 1 & - & 1 \\
FHA & 1 & 1 & 1 & - \\
Globin & - & - & - & 1 \\
Phytochrome & - & - & 2 & - \\
\hline
\end{tabular}

Of these TRT, TRR, TRRR and RTR geometry HYs dominate (39 proteins). These proteins are intriguing as it is likely that multiple routes of phospho transfer exist within each protein (presumably inter-dependently), potentially giving rise to sophisticated dynamic behaviour. One such example is RodK (Rasmussen et al., 2005), a TRRR protein that regulates the timing of fruiting body formation. All four domains of RodK are required for activity and each domain has a distinct phosphorylation-dependent function (Rasmussen et al., 2006). In the complex HYs of E. coli (ArcBA, BarA/UvrY, EvgAS and TorSR), signalling domains are located in sequential order $\mathrm{N}$ - to C-terminal, reflecting the direction of phosphoryl group transfer during signalling. This does not appear to be the case for the myxobacterial HYs, unless receiver-to-receiver and receiver-to-transmitter phosphotransfer also occurs.

Table 7. Domain architecture of myxobacterial HYs

Myxa, Myxococcus xanthus DK1622; Soce, Sorangium cellulosum So ce56; Stau, Stigmatella aurantiaca DW4/3-1; Ande, Anaeromyxobacter dehalogenans $2 \mathrm{CP}-\mathrm{C}$. $\mathrm{R}$, receiver domain; $\mathrm{T}$, transmitter domain.

\begin{tabular}{|lrrrrr|}
\hline & Myxa & Soce & Stau & Ande & Total \\
\hline TR & 14 & 24 & 19 & 10 & 67 \\
TRT & 1 & 5 & 6 & - & 12 \\
TRTR & - & 2 & 1 & - & 3 \\
TRTTRT & - & - & 1 & - & 1 \\
TRR & 5 & 1 & 5 & - & 11 \\
TRRR & 3 & 1 & 3 & - & 7 \\
TRRH & - & 1 & 1 & - & 2 \\
RT & 10 & 10 & 21 & 4 & 45 \\
RTR & 1 & 4 & 3 & 1 & 9 \\
RTRR & 1 & - & 1 & - & 2 \\
RRT & 1 & - & - & - & 1 \\
CheAY & 5 & 1 & 5 & 2 & 13 \\
Total & 41 & 49 & 66 & 17 & 173 \\
& & & & & \\
\hline
\end{tabular}

\section{Phylogenetic relationships and evolution of myxobacterial TCS proteins}

In order to assess homology relationships between myxobacterial TCS proteins and gain an understanding of the evolutionary events responsible for the contemporary set of proteins, phylogenetic trees were constructed for myxobacterial receiver and transmitter domains (see Methods). Fig. 5 is a phylogenetic distance tree derived from the DnaA protein sequences of the four myxobacteria and a selection of more distantly related organisms. The tree in Fig. 5 agrees with previously published molecular phylogenies (Sanford et al., 2002), and illustrates the evolutionary distance between the organisms under consideration. M. xanthus and St. aurantiaca are most closely related to each other, and together they are closer to A. dehalogenans than to So. cellulosum. TCS trees are available with the online version of this paper (Supplementary Figures S1, S2 and S3). For each leaf of the trees the domain architecture and gene organization is presented and leaves/clades are coloured according to the host organism. Splits were represented in colour/black if bootstrap support was obtained for that split $(>70 \%$ of bootstrapped trees contained that split using both neighbour-joining and parsimony methods), otherwise splits were coloured mid-grey if one method gave a bootstrap value $>70 \%$, or pale grey if neither method gave bootstrap support. The trees were then used to identify clades of homologues. Table 8 presents homology relationships for myxobacterial TCS proteins that have been experimentally characterized (based on the list presented in Whitworth \& Cock, 2007), using clades with bootstrap support $>50 \%$.

Fewer orthologues were identified with increasing evolutionary distance from M. xanthus (57 in St. aurantiaca, 25

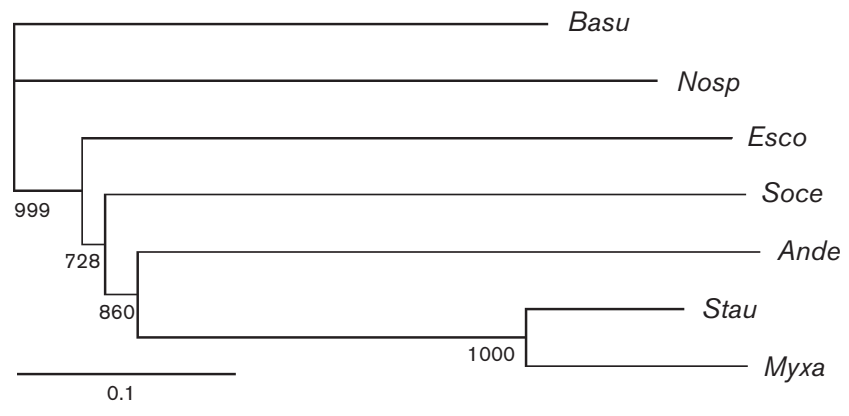

Fig. 5. Neighbour-joining phylogenetic tree of the DnaA protein of the four myxobacteria and selected non-myxobacterial bacteria. Numbers at nodes represent the number of times out of 1000 bootstrap replicates that the cluster defined by the node was monophyletic. Basu, Bacillus subtilis; Nosp, Nostoc sp.; Esco, Escherichia coli; Soce, Sorangium cellulosum; Ande, Anaeromyxobacter dehalogenans; Stau, Stigmatella aurantiaca; Myxa, Myxococcus xanthus. Bacillus subtilis was defined as the outgroup. 
Table 8. Experimentally characterized $M$. xanthus TCS proteins and their homologues in other myxobacterial genomes

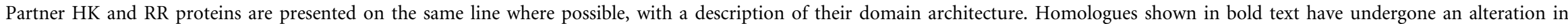
domain architecture relative to their M. xanthus counterparts, while those in italics have undergone a change in gene organization.

\begin{tabular}{|c|c|c|c|c|c|c|c|c|c|}
\hline \multicolumn{2}{|l|}{ Domain architecture } & \multicolumn{2}{|c|}{ M. xanthus } & \multicolumn{2}{|c|}{ St. aurantiaca } & \multicolumn{2}{|c|}{ A. dehalogenans } & \multicolumn{2}{|c|}{ So. cellulosum } \\
\hline HK & $\mathbf{R R}$ & HK & RR & HK & $\mathbf{R R}$ & HK & $\mathbf{R R}$ & HK & RR \\
\hline Hpt,HATPase,CheW,R & R,CheB & CheA3 & CheB & STIAU_1049 & STIAU_1047 & & Adeh_0614 & & \\
\hline Hpt,HATPase,CheW,R & $\mathrm{R}$ & CheA4 & CheY4 & STIAU_X008 & STIAU_4795 & & & & \\
\hline Hpt,HATPase,CheW & $\mathrm{R}$ & DifE & DifD & STIAU_8663 & STIAU_8664 & Adeh_4316 & Adeh_4317 & & \\
\hline \multirow[t]{3}{*}{ Hpt,HATPase,CheW,R } & R,CheB & FrzE & FrzG & STIAU_6834 & STIAU_6833 & Adeh_0613 & & & \\
\hline & $\mathrm{R}, \mathrm{R}$ & & FrzZ & & STIAU_6840 & & Adeh_0609 & & \\
\hline & $\mathrm{R}$, coiled-coil & & FrzS & & STIAU_6845 & & Adeh_2380 & & Sce5864 \\
\hline $\mathrm{T}$ & $\mathrm{R}, \mathrm{R}$ & RedC & RedD & STIAU_3439 & STIAU_3440 & & & $\begin{array}{l}\text { Sce5167, } \\
\text { Sce8798 }\end{array}$ & $\begin{array}{r}\text { Sce5166, } \\
\text { Sce } 8799\end{array}$ \\
\hline $\mathrm{T}$ & $\mathrm{R}$ & RedE & RedF & STIAU_3441 & STIAU_3442 & & & $\begin{array}{l}\text { Sce5168, } \\
\text { Sce8797 }\end{array}$ & Sce 8800 \\
\hline $\mathrm{T}, \mathrm{R}, \mathrm{R}, \mathrm{R}$ & $\mathrm{R}$ & RodK & MXAN_0732 & STIAU_5673 & STIAU_5674 & & & & \\
\hline PAS,PAS,T & $\mathrm{R}$ & TodK & DotR & STIAU_0573 & STIAU_6974 & & Adeh_0606 & & $\begin{array}{l}\text { Sce } 2424, \\
\text { Sce2206 }\end{array}$ \\
\hline \multirow[t]{6}{*}{$\mathrm{R}, \mathrm{T}$} & $\mathrm{R}$ & AsgA & MXAN_2671 & STIAU_4780 & STIAU_4782 & & & Sce6174 & \\
\hline & $\mathrm{R}$ & & RomR & & STIAU_0910 & & Adeh_2391 & & Sce0012 \\
\hline & $\mathrm{R}$, coiled-coil & & AglZ & & STIAU_7168 & & Adeh_2705 & & \\
\hline & R,GGDEF & & ActA & & & & & & \\
\hline & R,HTH_Xre & & DigR & & STIAU_6121 & & & & Sce0622 \\
\hline & R,HTH_LuxR & & FruA & & & & & & \\
\hline $\mathrm{T}$ & R,Trans_reg_C & PhoR1 & PhoP1 & STIAU_5393 & STIAU_5394 & & & & \\
\hline $\mathrm{T}$ & R,Trans_reg_C & PhoR2 & PhoP2 & STIAU_0112 & STIAU_X001 & & & & \\
\hline \multirow[t]{4}{*}{$\mathrm{T}$} & R,Trans_reg_C & PhoR3 & PhoP3 & STIAU_0427 & STIAU_X002 & & & & \\
\hline & R,Trans_reg_C & & PhoP4 & & STIAU_5390 & & & & \\
\hline & R,AAA_ATPase,HTH_Fis & & CrdA & & STIAU_1056 & & Adeh_3338 & & Sce1691 \\
\hline & R,AAA_ATPase,HTH_Fis & & $\mathrm{ActB}$ & & STIAU_5048 & & Adeh_2651 & & \\
\hline $\mathrm{T}$ & R,AAA_ATPase,HTH_Fis & FrgB & FrgC & STIAU_3782 & STIAU_3783 & & & & \\
\hline $\mathrm{T}$ & R,AAA_ATPase,HTH_Fis & MrpA & MrpB & STIAU_1020 & STIAU_1022 & & & & \\
\hline \multirow[t]{2}{*}{ PutP,PAS,T } & R,AAA_ATPase,HTH_Fis & MXAN_5852 & Nla1 & & & & Adeh_4250 & Sce2727 & Sce2692 \\
\hline & R,AAA_ATPase,HTH_Fis & & Nla4 & & STIAU_4539 & & Adeh_0688 & & Sce9222 \\
\hline HisKA & R,AAA_ATPase,HTH_Fis & MXAN_4043 & Nla6 & STIAU_6745 & STIAU_6743 & & Adeh_1555 & & \\
\hline $\mathrm{T}$ & R,AAA_ATPase,HTH_Fis & MXAN_5778 & $\mathrm{Nla} 23$ & STIAU_7821 & STIAU_7820 & Adeh_0638 & Adeh_0639 & & Sce2881 \\
\hline HAMP,T & R,AAA_ATPase,HTH_Fis & MXAN_7439 & $\mathrm{Nla} 24$ & & & & & & \\
\hline $\mathrm{T}$ & R,AAA_ATPase,HTH_Fis & MXAN_1166 & $\mathrm{Nla} 28$ & STIAU_3702 & STIAU_3701 & Adeh_0021 & & Sce0947 & \\
\hline PAS,T & R,AAA_ATPase,HTH_Fis & Pils & PilR & STIAU_7829 & STIAU_7828 & Adeh_0625 & Adeh_0626 & & \\
\hline HAMP,T & R,AAA_ATPase,HTH_Fis & SasS & SasR & STIAU_3603 & STIAU_3617 & Adeh_4246 & Adeh_4240 & & \\
\hline PAS,GAF,T & R,AAA_ATPase,HTH_Fis & SpdS & SpdR & STIAU_2431 & STIAU_2430 & Adeh_0418 & Adeh_0417 & & Sce1649 \\
\hline $\mathrm{R}, \mathrm{T}$ & R,AAA_ATPase,HTH_Fis & $\mathrm{HsfB}$ & HsfA & STIAU_6307 & STIAU_6308 & Adeh_3472 & Adeh_3471 & & \\
\hline R,GAF,GAF,T & & AsgD & & STIAU_0817 & & & & & \\
\hline
\end{tabular}




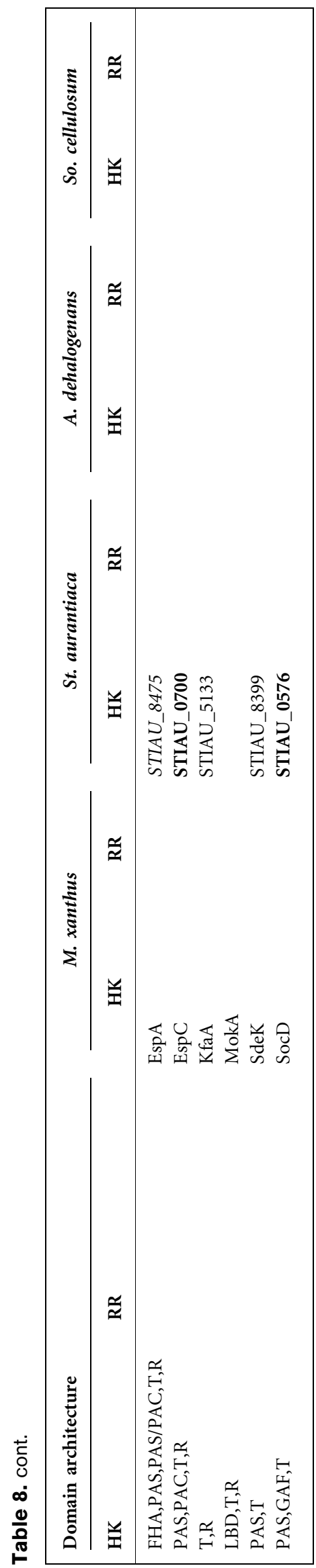

in A. dehalogenans, and 20 in So. cellulosum, respectively), presumably due to either sequence divergence or gene gain/ loss. Several changes in character were observed among the set of homologues. In 24 cases the gene organization of homologues had changed relative to the M. xanthus genes as a consequence of recruitment/loss of neighbouring TCS genes. Four duplications of characterized $M$. xanthus genes were also observed amongst their myxobacterial homologues, but only in So. cellulosum (three of the four red operon genes, and $\operatorname{dot} R$ ). Additionally, in seven cases the homologues presented in Table 8 also exhibited changes of domain architecture. In five cases the domain changes were recruitment/loss of an input domain within a HK gene. The sixth case is the A. dehalogenans FrzZ homologue, which lacks the first receiver domain of the M. xanthus and St. aurantiaca proteins, while the seventh case is the AsgA homologue of So. cellullosum, which has an AAA_ATPase,HTH_Fis domain instead of a transmitter domain.

Phylogenetic trees were also assessed in their entirety for evidence of gross genetic changes. This was achieved by identifying all clades of paired homologous domains from M. xanthus and St. aurantiaca (with strong bootstrap support, $>70 \%$ using both algorithms), and assessing whether there was any change in character between the two proteins. Particularly, we addressed changes in gene organization, gain/loss of input and output domains, and fusion/fission of TCS genes. In the majority of cases ( 85 protein pairs) we could identify no change in character between orthologous proteins; however, in many cases (25) input/output domains were missing from one orthologue, with changes of input domain (21 cases) far outnumbering changes in output domain (four cases). We identified 25 protein orthologues in which changes of gene organization had occurred, and a further three instances where adjacent TCS genes had apparently fused or split. Finally, we counted all clades in the trees composed solely of paralogous domains, which are suggestive of organism-specific domain duplications. The vast majority of duplicated domains came from So. cellulosum (52 paralogous domains), with large numbers also evident in the St. aurantiaca (28 domains) and $A$. dehalogenans (15 domains) genomes. Surprisingly, $M$. xanthus seems to have had relatively small numbers of domain duplications since splitting from the common ancestor (two paralogous domains, MXAN_0176 and MXAN_0706).

\section{DISCUSSION}

The myxobacteria as a group include organisms that possess the largest number of TCS genes of all prokaryotes, more even than would be expected for organisms with typically large genomes. This large regulatory potential correlates with an extremely sophisticated and complex lifestyle, characterized by cooperative multicellular development and metabolic diversity. But myxobacteria also 
seem to organize and utilize their TCS complement in unusual ways. An unusually large proportion of TCS genes are either orphaned or in complex gene clusters, suggesting more complex action than the typical TCS. Additionally, a disproportionate number of sensor kinases lack transmembrane helices, suggesting that they are responding to internal state changes. This is not particularly surprising, as most experimentally characterized TCSs of $M$. xanthus are known to be involved in regulating the gene transcription network governing fruiting body formation (Whitworth \& Cock, 2007).

Unfortunately, not enough is understood about TCS input domains to postulate signals to which myxobacterial TCSs respond; however, domain complements suggest a common mode of sensing, with St. aurantiaca particularly favouring GAF domains, and PAS/PAC being unusually common in myxobacterial proteins. More is known about TCS output domains, and a wide variety of such domains are found in the myxobacteria. Some are notably abundant, particularly AAA_ATPase,HTH_Fis domains, which are known to be used in large numbers to regulate fruiting and motility (Caberoy et al., 2003), but response regulators lacking any output domains are also abundant. Presumably these affect responses within the cell through proteinprotein interactions, or act as relays within complex phosphotransfer schemes. Such complicated phosphotransfer schemes appear to be encoded within several HYs, often found together in the genome, and possessing multiple transmitter and receiver domains. It has been argued that phosphorelays have evolved because they possess many points at which the phosphotransfer scheme can be regulated, or at which phosphoryl groups can be introduced into the signalling scheme (Burbulys et al., 1991). If this is the case, then the complex architectures of the myxobacterial HYs would suggest that these proteins act as important points for TCS signal integration, worthy of experimental focus.

In order to facilitate experimental analysis beyond the model myxobacterium $M$. xanthus, we have identified homologues of characterized $M$. xanthus proteins in the other myxobacterial genomes. Intriguingly, sets of orthologues seem to have been subjected to a large degree of gene rearrangements. It is tempting to speculate that the multiplicity of conserved domains among the TCS proteins has facilitated homologous recombination within evolving myxobacterial lineages, a hypothesis that requires many more myxobacterial genome sequences to test. Amongst sets of myxobacterial orthologues there are also a surprisingly large number of domain architecture changes, although it is intriguing that the majority of these (84\%) involve the gain/loss of input domains, while the minority affect output domains. This may suggest that the evolution of myxobacterial regulatory systems has progressed predominantly by changing which environmental cues are sensed, rather than by altering the responses generated within the cell. We also find a large degree of withinlineage domain duplications.
Another striking observation as a result of our analysis was the similarity of myxobacterial TCSs to those of cyanobacteria, particularly Anabaena and Nostoc. In terms of cytoplasmic sensing, lack of paired genes, dominance of GAF input domains, abundance of response regulators lacking output domains, and the presence of complex multi-domain HYs, the characteristics that make myxobacterial TCS regulation unusual, are also particularly characteristic of cyanobacteria.

The most remarkable features of myxobacterial biology are the social phenomena of fruiting body formation, sporulation and predation. It appears at first glance that multicellularity requires an increased regulatory complement relative to asocial organisms; however, it is intriguing that the increase in regulatory complexity in myxobacteria seems more profound for systems responding to internal stimuli rather than to extracellular events. Multicellular fruiting body formation has been shown to require just two inter-cellular signals (the diffusible A-signal and cellcontact mediated $\mathrm{C}$-signal). It may be the case that myxobacteria use their internal metabolism as an indicator of extracellular events, thus responding to environmental change indirectly. However, there is no convincing argument why that should necessarily be a requirement for multicellularity. In the endospore-forming bacteria such as Bacillus subtilis, there is no dramatic increase in TCS complexity over comparable non-spore formers, therefore spore formation per se does not require the level of regulatory complexity seen in the myxobacteria. Similarly, predatory bacteria such as Bdellovibrio bacteriovorus manage to predate without regulatory networks as large as those of the myxobacteria. Why then the increase in regulatory complexity observed during the evolution of multicellularity? We propose that evolution of multicellularity provides more of a challenge than just being able to interact with other cells within a population. Myxobacterial strains which defect or cheat during the developmental process have been characterized (Velicer et al., 2000) and it seems likely that the ability to protect the cooperative population from defectors/cheaters would require sophisticated regulatory mechanisms (Velicer, 2005). Similarly in the natural environment many myxobacterial strains co-exist (Vos \& Velicer, 2006), which presumably makes the nature of 'self' and 'self-recognition' important determinants of evolutionary fitness. Thus we propose that the complexity in TCS regulation of the myxobacteria may reflect 'hard-wired' regulatory systems that make the multicellular population robust to a changing social milieu. This conjecture suggests that differences in TCS regulation between myxobacterial species/strains may reflect modifications to the basic scheme for multicellular behaviour that provide some element of 'self' (or non-'self' incompatibility) to a population.

In summary, by categorizing the total set of TCS proteins from four myxobacterial genome sequences we have provided a framework for research into the TCSs of the myxobacteria. In addition to providing lists of gene sets and 
mapping homologues, the phylogenetic trees provided here should guide research into the TCSs of myxobacteria beyond M. xanthus, and possibly even direct experimental analysis of $M$. xanthus regulation through evolutionary arguments.

\section{ACKNOWLEDGEMENTS}

P. J.A.C. was funded by an EPSRC studentship through the MOAC Doctoral Training Centre. D.E.W. received funding from BBSRC grant BBD0039891. Computing resources were provided by the Centre for Scientific Computing at the University of Warwick. We wish to thank Rolf Müller (Universität des Saarlandes) for pre-release access to the Sorangium cellulosum genome sequence, and Andrew Millard for critical comments on the manuscript.

\section{REFERENCES}

Appleby, J. L., Parkin, J. S. \& Bourret, R. B. (1996). Signal transduction via the multi-step phosphorelay: not necessarily a road less traveled. Cell 86, 845-848.

Bijlsma, J. J. \& Groisman, E. A. (2003). Making informed decisions: regulatory interactions between two-component systems. Trends Microbiol 11, 359-366.

Burbulys, D., Trach, K. A. \& Hoch, J. A. (1991). The initiation of sporulation in Bacillus subtilis is controlled by a multicomponent phosphorelay. Cell 64, 545-552.

Caberoy, N. B., Welch, R. D., Jakobsen, J. S., Slater, S. C. \& Garza, A. G. (2003). Global mutational analysis of NtrC-like transcriptional activators in Myxococcus xanthus: identifying activator mutants defective for motility and fruiting body development. $J$ Bacteriol 185, 6083-6094.

Cock, P. J. A. \& Whitworth, D. E. (2007a). Evolution of gene overlaps: relative reading frame bias in prokaryotic two-component system genes. J Mol Evol 64, 457-462.

Cock, P. J. A. \& Whitworth, D. E. (2007b). Evolution of prokaryotic two-component system signaling pathways: gene fusions and fissions. Mol Biol Evol 24, 2355-2357.

Dawid, W. (2000). Biology and global distribution of myxobacteria in soils. FEMS Microbiol Rev 24, 403-427.

Ellehauge, E., Norregaard-Madsen, M. \& Søgaard-Andersen, L. (1998). The FruA signal transduction protein provides a checkpoint for the temporal co-ordination of inter-cellular signals in Myxococcus xanthus development. Mol Microbiol 30, 807-817.

Fabret, C., Feher, V. A. \& Hoch, J. A. (1999). Two-component signal transduction: how one organism sees its world. J Bacteriol 181, 1975-1983.

Felsenstein, J. (1989). PHYLIP - Phylogeny Inference Package (Version 3.2). Cladistics 5, 164-166.

Galperin, M. Y. (2005). A census of membrane-bound and intracellular signal transduction proteins in bacteria: bacterial IQ, extroverts and introverts. BMC Microbiol 5, 35.

Galperin, M. Y. (2006). Structural classification of bacterial response regulators: diversity of output domains and domain combinations. J Bacteriol 188, 4169-4182.

Gerth, K., Pradella, S., Perlova, O., Beyer, S. \& Muller, R. (2003). Myxobacteria: proficient producers of novel natural products with various biological activities - past and future biotechnological aspects with the focus on the genus Sorangium. J Biotechnol 106, 233-253.

Goldman, B. S., Nierman, W. C., Kaiser, D., Slater, S. C., Durkin, A. S., Eisen, J. A., Ronning, C. M., Barbazuk, W. B., Blanchard, M. \& other authors (2006). Evolution of sensory complexity recorded in a myxobacterial genome. Proc Natl Acad Sci U S A 103, 15200-15205.

Gronewald, T. M. A. \& Kaiser, D. (2001). The act operon controls the level and time of C-signal production for Myxococcus xanthus development. Mol Microbiol 40, 744-756.

Higgs, P. I., Cho, K., Whitworth, D. E., Evans, L. S. \& Zusman, D. R. (2005). Four unusual two-component signal transduction homologs, RedC to RedF, are necessary for timely development in Myxococcus xanthus. J Bacteriol 187, 8191-8195.

Hoch, J. A. \& Varughese, K. I. (2001). Keeping signals straight in phosphorelay signal transduction. J Bacteriol 183, 4941-4949.

Hutchings, M. I., Hoskisson, P. A., Chandra, G. \& Buttner, M. J. (2004). Sensing and responding to extracellular signals? Analysis of the sensor kinases and response regulators of Streptomyces coelicolor A3(2). Microbiology 150, 2795-2806.

Jelsbak, L., Givskov, M. \& Kaiser, D. (2005). Enhancer-binding proteins with a forkhead-associated domain and the $\sigma^{54}$ regulon in Myxococcus xanthus fruiting body development. Proc Natl Acad Sci U S A 102, 3010-3015.

Kaiser, D. (2004). Signaling in myxobacteria. Annu Rev Microbiol 58, 75-98.

Mailund, T. \& Pedersen, C. N. S. (2004). QuickJoin - fast neighbourjoining tree reconstruction. Bioinformatics 20, 3261-3262.

Mizuno, T. (1997). Compilation of all genes encoding two-component phosphotransfer signal transducers in the genome of Escherichia coli. DNA Res 4, 161-168.

Mizuno, T., Kaneko, T. \& Tabata, S. (1996). Compilation of all genes encoding bacterial two-component signal transducers in the genome of the cyanobacterium, Synechocystis sp. strain PCC 6803. DNA Res 3, 407-414.

Neumann, B., Pospiech, A. \& Schairer, H. U. (1993). A physical and genetic map of the Stigmatella aurantiaca DW4/3.1 chromosome. Mol Microbiol 10, 1087-1099.

Parkinson, J. S. \& Kofoid, E. C. (1992). Communication modules in bacterial signaling proteins. Annu Rev Genet 26, 71-112.

Peña-Sandoval, G. R., Kwon, O. \& Georgellis, D. (2005). Requirement of the receiver and phosphotransfer domains of ArcB for efficient dephosphorylation of phosphorylated ArcA in vivo. J Bacteriol 187, 3267-3272.

Pollack, J. S. \& Singer, M. (2001). SdeK, a histidine kinase required for Myxococcus xanthus development. J Bacteriol 183, 3589-3596.

Pradella, S., Hans, A., Sproer, C., Reichenbach, H., Gerth, K. \& Beyer, S. (2002). Characterisation, genome size and genetic manipulation of the myxobacterium Sorangium cellulosum So ce56. Arch Microbiol 178, 484-492.

Rasmussen, A. A., Porter, S. L., Armitage, J. P. \& Sogaard-Andersen, L. (2005). Coupling of multicellular morphogenesis and cellular differentiation by an unusual hybrid histidine protein kinase in Myxococcus xanthus. Mol Microbiol 56, 1358-1372.

Rasmussen, A. A., Wegener-Feldbrugge, S., Porter, S. L., Armitage, J. P. \& Sogaard-Andersen, L. (2006). Four signalling domains in the hybrid histidine protein kinase RodK of Myxococcus xanthus are required for activity. Mol Microbiol 60, 525-534.

Sanford, R. A., Cole, J. R. \& Tiedje, J. M. (2002). Characterization and description of Anaeromyxobacter dehalogenans gen. nov., sp. nov., an aryl-halorespiring facultative anaerobic myxobacterium. Appl Environ Microbiol 68, 893-900.

Schneiker, S., Perlova, O., Kaiser, O., Gerth, K., Alici, A., Altmeyer, M. O., Bartels, D., Bekel, T., Beyer, S. \& other authors (2007). Complete genome sequence of the myxobacterium Sorangium cellulosum. Nat Biotechnol 25, 1281-1289. 
Sonnhammer, E. L., Eddy, S. R. \& Durbin, R. (1997). Pfam: a comprehensive database of protein domain families based on seed alignments. Proteins 28, 405-420.

Sonnhammer, E. L., von Heijne, G. \& Krogh, A. (1998). A hidden Markov model for predicting transmembrane helices in protein sequences. Proc Int Conf Intell Syst Mol Biol 6, 175-182.

Thompson, J. D., Higgins, D. G. \& Gibson, T. J. (1994). CLUSTAL W: improving the sensitivity of progressive multiple sequence alignment through sequence weighting, position-specific gap penalties and weight matrix choice. Nucleic Acids Res 22, 4673-4680.

Trudeau, K. G., Ward, M. J. \& Zusman, D. R. (1996). Identification and characterization of FrzZ, a novel response regulator necessary for swarming and fruiting-body formation in Myxococcus xanthus. Mol Microbiol 20, 645-655.

Velicer, G. J. (2005). Evolution of cooperation: does selfishness restraint lie within? Curr Biol 15, R173-R175.

Velicer, G. J., Kroos, L. \& Lenski, R. E. (2000). Developmental cheating in the social bacterium Myxococcus xanthus. Nature 404, 598-601.
Vos, M. \& Velicer, G. J. (2006). Genetic population structure of the soil bacterium Myxococcus xanthus at the centimeter scale. Appl Environ Microbiol 72, 3615-3625.

Ward, M. J., Lew, H. \& Zusman, D. R. (2000). Social motility in Myxococcus xanthus requires FrzS, a protein with an extensive coiledcoil domain. Mol Microbiol 37, 1357-1371.

West, A. H. \& Stock, A. M. (2001). Histidine kinases and response regulators in two-component signaling systems. Trends Biochem Sci 26, 369-376.

Whitworth, D. E. (2007). Myxobacteria: Multicellularity and Differentiation. Washington, DC: American Society for Microbiology.

Whitworth, D. E. \& Cock, P. J. A. (2007). Myxobacterial two-component systems. In Myxobacteria: Multicellularity and Differentiation, pp. 169189. Edited by D. E. Whitworth. Washington, DC: American Society for Microbiology.

Edited by: D. W. Ussery 